\title{
Aa. Vv., «Cahiers de Recherches Médiévales», n. 14
}

\section{Maria Colombo Timelli}

\section{(2) OpenEdition}

\section{Journals}

\section{Édition électronique}

URL : http://journals.openedition.org/studifrancesi/8165

DOI : 10.4000/studifrancesi.8165

ISSN : 2427-5856

\section{Éditeur}

Rosenberg \& Sellier

\section{Édition imprimée}

Date de publication : 1 mai 2009

Pagination : 141-142

ISSN : 0039-2944

\section{Référence électronique}

Maria Colombo Timelli, « Aa. VV., «Cahiers de Recherches Médiévales», n. 14 », Studi Francesi [En ligne], 157 (LIII | I) | 2009, mis en ligne le 30 novembre 2015, consulté le 12 janvier 2021. URL : http:// journals.openedition.org/studifrancesi/8165; DOI : https://doi.org/10.4000/studifrancesi.8165

\section{Ce document a été généré automatiquement le 12 janvier 2021.}

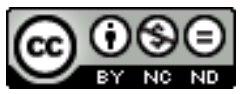

Studi Francesi è distribuita con Licenza Creative Commons Attribuzione - Non commerciale - Non opere derivate 4.0 Internazionale. 


\title{
Aa. Vv., «Cahiers de Recherches Médiévales», n. 14
}

\author{
Maria Colombo Timelli
}

\section{RÉFÉRENCE}

«Cahiers de Recherches Médiévales», n. 14, 2007.

1 La première partie de cette issue, sous la direction de William Kléber, concerne L'héritage de Chrétien de Troyes.

2 кеith BUSBy (Post-Chrétien Verse Romance: The Manuscript Context, pp. 11-24) reprend ici en partie le chapitre $\mathrm{V}$ de son livre Codex and Context (2002), où il montre l'intérêt d'une étude qui prenne en compte le contexte de transmission des œuvres (surtout le contenu des manuscrits et leur iconographie): il examine en particulier le ms. 472 de Chantilly, le ms. unique du Roman de Silence (Nottingham UL, Mi.LM.6), trois des cinq manuscrits qui nous ont transmis Meraugis de Portlesguez, les mss. BnF fr. 12603 et fr. 2168. L'étude du contenu des témoins permet en particulier de resituer les romans arthuriens tardifs dans le cadre plus large de la production narrative en vers du XIII ${ }^{\mathrm{e}}$ siècle (romans non-arthuriens, chansons de geste, récits brefs, lais et fabliaux).

3 Douglas KELLY (La conjointure de l'anomalie et du stéréotype: un modèle de l'invention dans les romans arthuriens en vers, pp. 25-39) compare les modalités de la description physique, morale et vestimentaire, dans un corpus qui réunit les romans de Chrétien et ceux de ses émules du xiII ${ }^{\mathrm{e}}$ siècle, en tenant compte également de la tradition scolastique illustrée dans les arts poétiques contemporains. Il peut ainsi reconnaître une sorte de modèle commun, où la description stéréotypée s'accompagne parfois d'une anomalie (il en est ainsi par exemple pour Enide, dont la beauté et la noblesse contrastent avec la pauvreté initiale de ses vêtements, ou pour Erec, blandus amator uxoris juste après leur mariage), anomalie que les aventures successives vont corriger. C'est ainsi que le caractère idéal des personnages est garanti à la fin de leur parcours chevaleresque. 
4 Le propos de Norris J. LACY (Arthur's Character and Reputation in "Yder", pp. 41-48) est de nuancer le jugement que la critique a porté jusqu'ici sur le personnage d'Arthur dans ce roman du début $\mathrm{du} \mathrm{XIII}^{\mathrm{e}}$ siècle. En relisant quelques passages représentatifs, il démontre que le roi est une figure complexe: jaloux certes, souvent inadéquat à son rôle, son comportement a des conséquences négatives sur sa cour, qui perd sa charge symbolique et idéale.

5 Christine FERLAMPIN-ACHER (La Table Ronde dans les "Merveilles de Rigomer", pp. 49-59) analyse trois aspects essentiels de ce roman: (1) la Table Ronde, qui perd ici son rôle premier de centre symbolique du monde arthurien réunissant un nombre précis bien que changeant de chevaliers (2) l'omniprésence, presque en contrepoids, da la table (thèmes de la nourriture et de la cuisine), qui semble parodier le motif du Graal (3) la présence d'une 'mesnie Arthur', qui rappelle évidemment la 'mesnie Hallequin' et qui transforme l'ancien monde de Chrétien en un groupe de revenants. C'est sur la base de ces éléments que C.F.-A. pense pouvoir dater les Merveilles de c. 1250 plutôt que de la fin du XII ${ }^{\mathrm{e}}$ siècle.

Peggy MCCRACKEN (Maternity and Chivalry after Chrétien: the Case of Lot's Wife, pp. 75-85) constate que la mère de Gauvain, dont le nom n'est même pas fixé dans la tradition arthurienne, demeure rattachée à des transgressions: dans le Perlesvaus on rappelle brièvement la naissance de Gauvain et l'abandon de l'enfant, le Lancelot en prose raconte sa relation incestueuse avec le roi Arthur et la naissance de Mordret, dans le Tristan en prose enfin on lit ses amours avec Lamorat, fils de l'assassin de son mari. Une lecture intertextuelle permet de comprendre que la liberté des narrateurs dépend en grande partie du silence qui entoure ce personnage.

7 Donald MADDOX (Sens and "conjointure armoriale" dans le "Lancelot" propre, pp. 87-99) reconnaît dans la série de onze écus qu'arbore Lancelot quelques caractéristiques saillantes: d'une part, l'insertion en est due à des personnages féminins, d'autre part ils correspondent à un parcours chromatique allant du blanc au rouge au noir. Qui plus est, cet intérêt pour les couleurs héraldiques caractérise en propre cette section du roman, et marque les deux parties, histoire privée et histoire collective, qui la composent.

8 La seconde partie du volume, coordonnée par Silvère Menegaldo, porte sur Girart d'Amiens, l'auteur le plus prolifique du Moyen Âge, qui a laissé quelque 70000 vers pour trois poèmes, sur lequel manque toujours un travail de synthèse. On trouvera ici quatre contributions. Dans la première, Damien DE CARNÉ (Escanor dans son roman, pp. 153-175) s'attache à montrer la complexité du personnage d'Escanor, construit à partir de modèles antérieurs, la Troisième Continuation de Gerard de Montreuil et l'Atre périlleux surtout, qui révèlent quelque chose de la culture littéraire de Girart d'Amiens. Deuxièmement est reproduite une partie de la thèse d'Antoinette SALY (1977, Girart d'Amiens romancier. La composition de "Meliacin", pp. 177-188) et notamment les pages consacrées à la composition de ce roman, qui a toujours souffert de la comparaison avec Cleomadés d'Adenet le Roi et avec sa source, un conte tiré des Mille et une nuits. Alain CORBELlARI (Le "Charlemagne" de Girart d'Amiens et la tradition épique française, pp. 189-199) consacre ses remarques à cette longue biographie de l'empereur (23000 vers environ), dans laquelle Girart mélange des sources épiques et historiques. Pour le même ouvrage, Daniel MÉTRAUX (Le Charlemagne de Girart d'Amiens: vers un empereur modèle, pp. 201-207) met l'accent sur le deuxième livre et sur le portrait de Charlemagne qui en ressort: personnage vivant, économiste de valeur, grand 
intellectuel, en harmonie avec la figure historique mais aussi avec les idéaux de l'époque de Girart et de son dédicataire Charles de Valois.

9 La section Études christiniennes comprend une contribution de Karen GREEN: Could Christine de Pizan be the author of the "Advis à Isabelle de Bavière", BNF MS fr. 1223?, pp. 211-229. Elle discute les différents arguments qui permettent de soutenir et de contester la paternité de l'œuvre; après avoir proposé une datation ante 1429 (couronnement de Charles VII), elle confirme l'attribution sur la base surtout de la présence de thèmes communs avec d'autres œuvres de Christine.

10 Enfin, sont réunies sous l'intitulé de Varia deux articles que leur sujet n'éloigne pas trop l'un de l'autre. Dans le premier, Aurélie BARRE (Le renard de Rutebeuf, pp. 253-266) montre comment le renard est au centre de la poésie de Rutebeuf: animal fourbe et rusé dans la tradition des Bestiaires, il devient l'image même des hypocrites et des mendiants; de surcroît, la renardie marque aussi l'écriture du poète, qui se masque et se déguise derrière les jeux de la langue et de l'écriture. Dans l'autre, Jean-François KOSTA-THÉFAINE reconnaît l'importance des Poissons dans les poésies d'Eustache Deschamps (pp. 267-280) tant sur le plan statistique (relevés des désignations et des occurrences) que sur celui des fonctions que le poète peut leur attribuer: rattachés au régime quotidien, ils permettent à Deschamps d'exprimer ses propres goûts et sa préférence pour la France et sa cuisine, ainsi que de renvoyer à quelques détails historiques. 